\title{
Simulation Studies for Ion Beam Extraction Systems
}

\author{
M.M. Abdelrahman and S.G. Zakhary \\ Accelerators \& Ion Sources Department, Nuclear Research Center, AEA \\ P.O. Box: 13759, Inchas, Atomic Energy, Cairo, Egypt \\ (Received on 27 Dezember, 2008)
}

\begin{abstract}
The characteristics of the ion beam extracted from an ion sources were investigated using computer code SIMION 3 D Version 7.0. It has been used to evaluate the extraction system in order to produce an ion beam with high current and low beam emittance. The results show that the shape of the extraction electrode plays an important role in ion beam formation. Comparison has been made between two extraction systems, Pierce extraction electrode and spherical extraction electrode. The results show that the spherical extraction system yields ion extraction beam with lower emittance and radius than that the Pierce system. The simulation can provide the basis for optimizing the extraction system and the acceleration gap for ion source.
\end{abstract}

Keywords: Ion Beam Emittance, Plasma Meniscus, Space Charge, Ion Trajectories.

\section{INTRODUCTION}

The extraction of ions and ion beam formation process seem to be one of the most crucial points in ion implantation, electromagnetic separation and plasma diagnostics [1]. In order to extract ion beam from the appropriate ion source, an arrangement of carefully designed electrode must be used. This electrode must create the proper configuration of the electric field at the surface of the ion source and along the acceleration of the ion beam region [2]. The surface which forms the source of ions can be either of fixed geometry (surface ionization and field ion sources) or it can be the boundary of the plasma (plasma ion sources), in which the shape of the surface is fluid depending on the current density, ion supply rate and the applied electric field. The design of an extraction system must take into account the nature of this system and must initiate the ion beam as free of aberrations as possible. Pierce solved the problem of extracting an absolute parallel beam analytically for electrons. The law found by Pierce was not suited for the extraction of ions from plasma, especially of high current ion sources for the following reasons [3]:

Mechanical and power load, where the outlet electrode is not infinitely thin, the plasma potential is not exactly the same as the potential of the electrode, the plasma cannot deliver the same current density all over the aperture of the electrode because the plasma density decreases near the electrode and the equipotential surface near the second electrode is not planar, but curved outward.

The extraction of ions can be classified into two categories: (a) directly from the source plasma, which remains confined in the space where the ions are created, e.g. low current ion sources $\left(10^{10}\right.$ to $10^{12}$ ions $\left./ \mathrm{cm}^{3}\right)$. (b) From a plasma which has diffused out from the source through anode aperture (Sources of plasma density $10^{12}$ to $10^{14}$ ions $/ \mathrm{cm}^{3}$ ). In the presence of space charge, the electric field acting on an ion beam is $[2,4]$ :

$$
E_{r}=\frac{q}{2 \pi \varepsilon_{0} r}=\frac{I_{0}}{2 \pi \varepsilon_{0}{ }_{0} r}
$$

where $q$ is the charge of the beam per unit length within radius $r$ and $q=\frac{I_{0}}{0}$ where $I_{0}$ is the total beam current, 0 is the

Electronic address: moustafa82003@yahoo.com axial ion velocity $=((2 e / m) V)^{1 / 2}$ where $V$ is the extraction voltage and $\varepsilon 0$ is the permittivity of free space.

Equation (1) becomes $E_{r}=\frac{I_{o}}{2 \pi \varepsilon_{o} r(2 e / m)^{1 / 2} V^{1 / 2}}=\frac{I_{o}}{A r V^{1 / 2}}$

where $A=2 \pi \varepsilon_{o}(2 e / m)^{1 / 2}$

In this work, the simulation process using SIMION computer program has been used to study the influence of the cathode shape [Pierce electrode, spherical electrode with cylindrical inside canal, spherical electrode with Pierce inside canal (used in ion the beam extraction system)] on both beam diameter and beam emittance. The simulation process is made with varying extraction gap width, extraction voltage and space charge effect (ion beam current).

\section{Theoretical Treatment}

Pierce Extraction Electrode

The ions in the extraction electrode aperture are normally considered to be a lens with low aberrations. However in practice it has a thickness and spherical aberration arises. Also, in the simple analysis dealing with the ion optics, it was assumed that the ions emitted from a spherical or cylindrical surface follow radial paths under the influence of electric field which has only radial component. However, it has a component normal to the interface between the ion and the vacuum region. To achieve zero normal component of the electric field, the cathode should have a suitable shape by forming Pierce electrode geometry [6]. Pierce analysis showed that the potential outside.

The potential is given by [7]:

$$
V=A\left(z^{2}+y^{2}\right)^{2 / 3} \cos \left(\frac{4}{3} \tan ^{-1} \frac{y}{z}\right)
$$

It is seen from equation 3 that the zero equipotential occurs along a line passing through $z=0$ and making an angle equal to $(3 / 4)(\pi / 2)=67^{0} 1 / 2$ with the beam edge as shown in Fig. (1). 


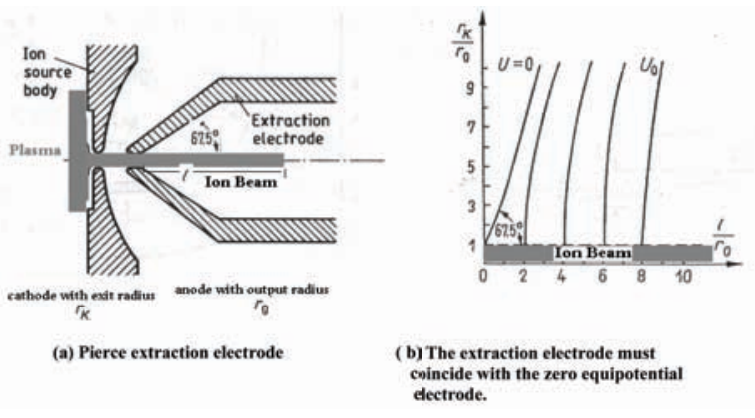

FIG. 1: Pierce extraction electrode.

\section{Influence of Spherical Extraction Electrode on the Beam Optics}

In the spherical extraction system shown in Fig. (2), the cathode (plasma emitting ion beam) and anode are spherical segments referenced to the same center. They have radii of curvature $\rho_{c}$ and $\rho_{a}$. Poisson equation has the following form for a spherically symmetric potential [8]:

$$
\frac{1}{r^{2}} \frac{d}{d r}\left(r^{2} \frac{d V}{d r}\right)=-\frac{\rho}{\varepsilon_{0}}
$$

where $\rho$ is the space charge density of the ions and related to the ion velocity by:

$\rho=-\frac{I}{4 \pi r^{2} v}$, where $v$ is the ion velocity, $v=\sqrt{\frac{2 e V}{m}}$

By substitution, Eq. (4) will become:

$$
\frac{d}{d r}\left(r^{2} \frac{d V}{d r}\right)=\frac{I}{4 \pi \varepsilon_{0}} \sqrt{\frac{m}{2 e V}}
$$

The solution which gives the perveance of a flow of spherical ion beam [8]:

$$
\frac{I_{\text {sphere }}}{V_{0}^{3 / 2}}=\frac{4 \varepsilon_{0}}{9} \sqrt{\frac{2 e}{m}} \frac{4 \pi}{\left[\alpha\left(\rho_{a} / \rho_{c}\right)\right]^{2}}
$$

The quantity $\alpha\left(\rho_{a} / \rho_{c}\right)$ is the Langmuir function for converging flow and it depends on the radius of curvature of the cathode and the anode [9].

The perveance of the ion beam equals the expression of equation 5 multiplied by the ratio of the cathode area $A_{c}$ to the area of the fall sphere $4 \pi \rho_{c}^{2}$ and the gas perveance is:

$$
\frac{I}{V_{0}^{3 / 2}}=\frac{4 \varepsilon_{0}}{9} \sqrt{\frac{2 e}{m}} \frac{\sin ^{2} \theta / 2}{\alpha^{2}\left(\rho_{a} / \rho_{c}\right)^{2}}
$$

Equation 7 can be rewritten as:

$$
P=\frac{I}{V_{0}^{3 / 2}}=\frac{4 \varepsilon_{0}}{9} \sqrt{\frac{2 e}{m}} \frac{1-\cos \theta}{2 \alpha^{2}}
$$

$\left[P=\frac{14.7}{\alpha^{2}}(1-\cos \theta)\left(\right.\right.$ for electrons), $P=\frac{0.34}{\alpha^{2}}(1-\cos \theta)$ (for proton) Amp/ (Volt) $\left.)^{3 / 2}\right]$.

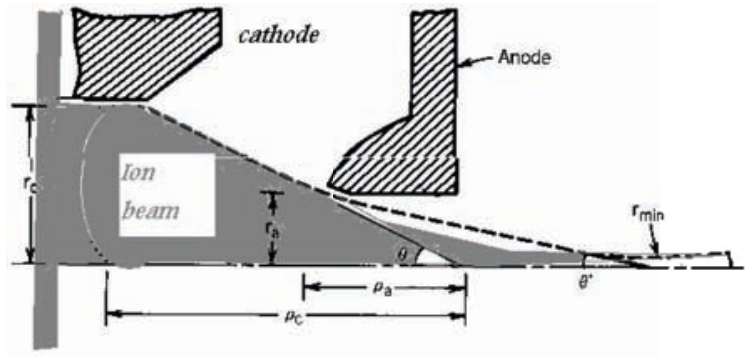

FIG. 2: Converging gun geometry for a beam with moderate perveance [10].

It follows that the quantity of $P$ of the (electron or ion) gun depends on the geometry of the extractor $\left(\theta, \rho_{c} / \rho_{a}\right)$. It is easy to conclude from eq. 8 that the perveance increases as the angle of divergence $\theta$ and the geometry $\rho_{c} / \rho_{a}$ of the extractor increase. The beam emerging from the aperture of a converging gun usually has strong space charge forces and low emittance. The minimum beam radius in terms of the envelope angle and beam perveance at the anode is [11]:

$$
\frac{\mathrm{r}_{\min }}{r_{a}}=e^{\frac{-3.3 \times 10^{-5} 5_{\theta} / 2}{\left(I / V_{0}^{3 / 2}\right)}}
$$

where

$$
\theta^{\prime}=\theta\left[1-\rho_{c} / 4\left(\rho_{c}-\rho_{a}\right)\right]
$$

\section{The Beam Emittance}

Liouville's theorem states that the motion of the charged particles under the action of conservative fields is such that the local number density in the six-dimensional phase space is a conserved quantity. If the transverse components of motion of a group of particles are mutually independent in space, they are also independent in the orthogonal phase space planes $\left(x, p_{x}\right),\left(y, p_{y}\right)$, and $\left(z, p_{z}\right)$ and the corresponding phase space areas are separately conserved. The transverse phase space areas are proportional to the emittances of the beam which are consequently also conserved. At a certain position on the $\mathrm{z}$-axis (momentum component $p_{z}$ approximately equals the total particle momentum $p$ ), we consider ions which pass through $(x, y)$ related to $r$, if cylindrical symmetry can be assumed [14]:

$$
x^{\prime}=\frac{p_{x}}{p_{z}} \simeq \frac{p_{x}}{p}, y^{\prime} \simeq \frac{p_{y}}{p}, r^{\prime} \simeq \frac{p_{r}}{p},
$$

and then,

$$
\varepsilon_{x}=\frac{1}{\pi} A\left(x, x^{\prime}\right), \varepsilon_{y}=\frac{1}{\pi} A\left(y, y^{\prime}\right), \varepsilon_{r}=\frac{1}{\pi} A\left(r, r^{\prime}\right),
$$

where $\varepsilon \mathrm{x}, \varepsilon_{y}$ and $\varepsilon \mathrm{r}$ are the emittances in $x-x^{\prime}, y-y^{\prime}$ and $r-r^{\prime}$ subspaces, respectively. The values A denote the areas within contours, where for all $\mathrm{x}$ the corresponding limits of $x^{\prime}$, for all $\mathrm{y}$ the corresponding limits of $\mathrm{y} /$ and for all $\mathrm{r}$ the corresponding limits of $r^{\prime}$ are plotted. These areas of $A\left(x, x^{\prime}\right)$, $A\left(y, y^{\prime}\right)$ and $A\left(r, r^{\prime}\right)$ represent two dimensional phase space areas and remain constant along a drift region (Fig. 3.2). For 


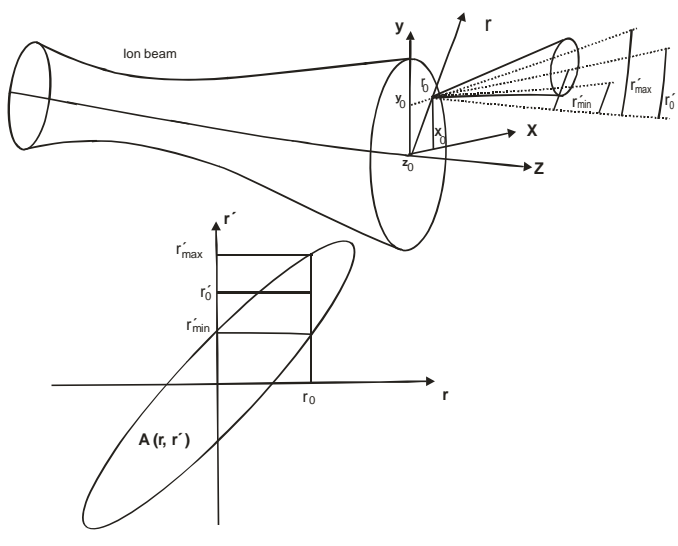

FIG. 3: Concept of beam emittance [11].

an accelerating field, the beam emittance can be defined as the normalised emittance (beam emittance multiplied with the respective momentum); these emittances are constants of the particle movement: ]

$$
\varepsilon_{n}=\frac{p}{m_{i} c} \varepsilon=\varepsilon
$$

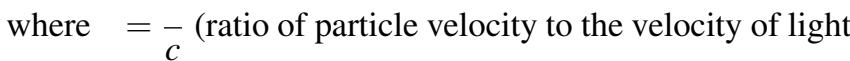
in vacuum), and $=(1-2)^{-1 / 2}$.

Another figure of merit used for characterizing the ion beam quality is the mean brightness, $\mathrm{B}$, which is defined in terms of the emittance $\varepsilon\left(r, r^{\prime}\right)$ as [11]:

$$
B=\frac{I}{\frac{\pi^{2}}{2}(\varepsilon(r, r))^{2}}=\frac{2 I}{\pi^{2} \varepsilon_{r}^{2}} .
$$

\section{Results And Discussion}

\section{Simulation Process}

SIMION 3 D Version $7.0[12,13]$ is a software package primarily used to calculate electric fields and the trajectories of charged particles in those fields when given a configuration of electrodes with voltages and particle initial conditions, including optional RF (quasistatic), magnetic field, and collisional effects. This program provides extensive supporting functionality in geometry definition, user programming, data recording, and visualization.. In general, the fundamental steps for simulating the properties of a model extraction system are to define the physical and electrical boundaries of the electrodes. SIMION defines the ions that make up the beam, selects output data to be recorded and simulates ion accelerated trajectories through the extraction system. Each electrode of this extraction system is separately designed using a potential array. Such a potential array is a two or three dimensional array of points, consisting of a collection of equally spaced points forming a rectangular grid. Points in the potential array will be bound within a certain shape creating an electrode or non-electrode. Using a finite difference method, SIMION takes the potentials of the electrode points to calculate the potential at the non-electrode points. Once all electrodes are designed and defined within a potential array, SIMION solves Laplace's equation:

$$
{ }^{2} V=0
$$

SIMION uses a highly modified $4^{\text {th }}$ order Runge-kutta technique for modelling the ion trajectories. Ion trajectories are a result of electrostatic and space charge repulsion forces on the basis of the current position and velocity of the ions. These forces are then used to compute the current ion acceleration and used by numerical integration techniques to predict the position and velocity of the ion at the next time step. Electrostatic forces are initially computed in terms of volts per grid unit. As the ion progresses through the potential array it moves from one square of grid points into another. SIMION automatically generates a small 16 point array that represents the current for four grid points and the 12 grid points around it. The values of these grid points are determined by symmetry assumptions and grid point location. The potential at each point is normally calculated via linear interpolation using four grid points bounding the grid square it falls in. When an ion is outside electrostatic instances, SIMION looks both directions along its current trajectory path for the closest electrostatic instance of intersection in both directions. If the present ion trajectory intersects electrostatic instances in both directions, SIMION will determine the potentials at the points of intersection and estimate the resulting electrostatic acceleration assuming a linear gradient.

Simulation of the singly charged ion trajectories for a plasma concave of curvature $4 \mathrm{~mm}$ was studied with and without space charge effect using two types of extraction systems using SIMION computer program. The plasma-electrode was considered as the plasma emission surface, where the ions are starting to fly away. Flying of ions will be individually and the number of flying ions were 500. The simulation process was carried out at an assumption of a constant plasma density. The voltage applied to the plasma electrode was $+5 \mathrm{kV}$. The voltage applied to the extraction electrode was varied and optimized to accomplish the suitable ion trajectories without hitting the extraction electrode. In this work, beam simulation is carried out to reveal the influence of the cathode shape on the beam emittance and beam radius. Three cathodes of different shapes are used. Fig. (4) (a, b, c) shows the beam simulation with the cathode has the form of Pierce (Fig. 4a), spherical without cylindrical canal (Fig. 4b) spherical with Pierce inside canal (Fig. 4c).

The increase of the extraction voltage affects decrease of the beam emittance (Figure (5)) and this can be explained by the decrease of the angle of the beam divergence which is found to be [14]:

$$
\theta=\sqrt{\frac{K T i}{e V}}
$$

where $q$ is the angle of beam divergence, For small angle tan $q=q=r^{\prime}, K T i 4$ is the transverse kinetic energy ( $K$ is the Planck's constant), Ti4 is the ion temperature and $e V$ is the axial accelerating energy.

Figure (5) shows the influence of the shape of inside canal on the bam emittance. The lower beam emittance is found when spherical with inside canal in the form of Pierce shape is 

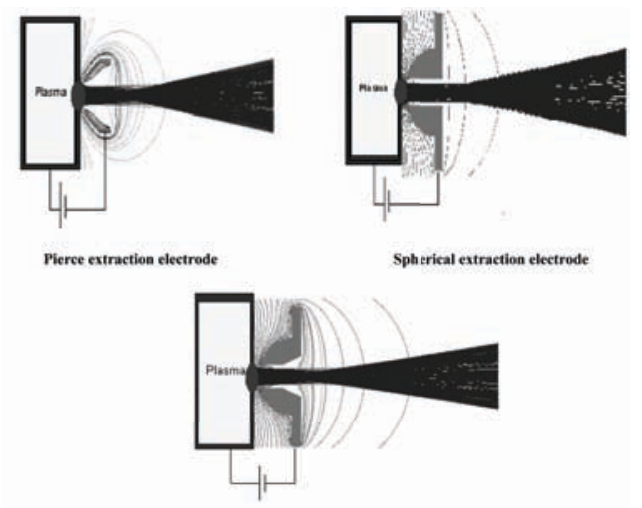

FIG. 4: Different cathode shapes.

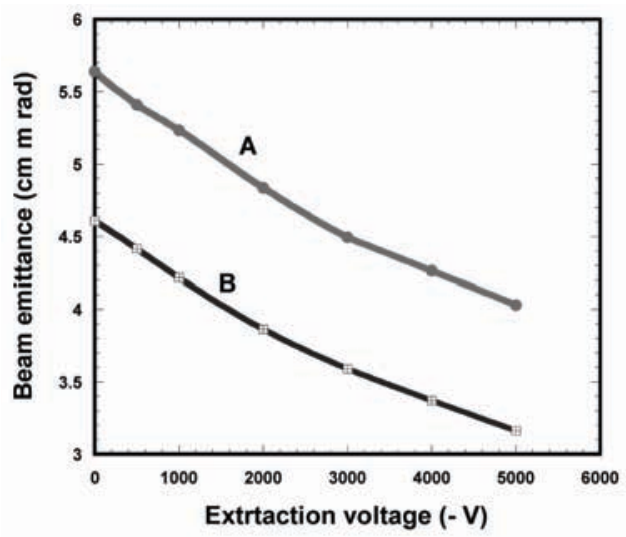

FIG. 5: Influence of the extraction voltage on the beam emittance: A - Spherical with cylindrical inside canal.

B - Spherical with inside canal in the form of Pierce shape.

used than that of cylindrical inside canal. The spherical shape of the cathode collimates the beam at the entrance while the Pierce inside canal decreases the space charge effect.

Influence of the applied negative voltage on both beam emittance and beam diameter for the two extraction systems has been studied (Fig. 6). It was found that, an increase of the applying negative voltage on the extraction electrode is accompanied by a decrease for both the beam emittance and beam diameter of the two types of extraction systems. Both the beam emittance and beam diameter for a spherical extraction system was found to be smaller than for a Pierce one.

The influence of the distance between the plasma-electrode and extraction electrode (extraction gap width) on the ion beam envelope was investigated for a concave meniscus with $4 \mathrm{~mm}$ curvature radius (Fig. 7). In these calculations, the voltage applied to the plasma electrode $V_{\text {plasma }}=+5 \mathrm{kV} 44$, The voltage applied to the extraction electrode was fixed to $V_{\text {extr }}=-4000 \mathrm{~V}$, which showing the ion beam envelope fully passed through the extraction region. Simulation of singly charged ions at different geometrical distances was achieved. It was found that an optimum extraction gap width at $3 \mathrm{~mm}$ for the spherical extraction system and at $2 \mathrm{~mm}$ for Pierce extraction system. At this distance, the ion beam envelope was fully passed through the extraction region.

The space charge is only considered in the gap between

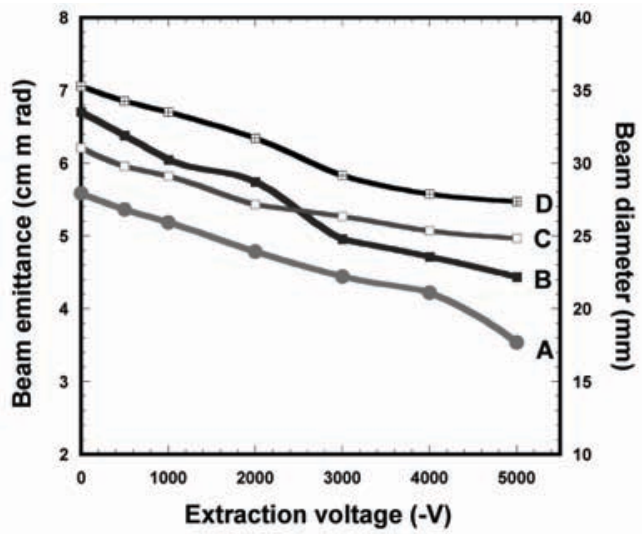

FIG. 6: Influence of the extraction voltage on both beam emittance (A/spherical, B/Pierce) and beam diameter (C/spherical, D/ Pierce).

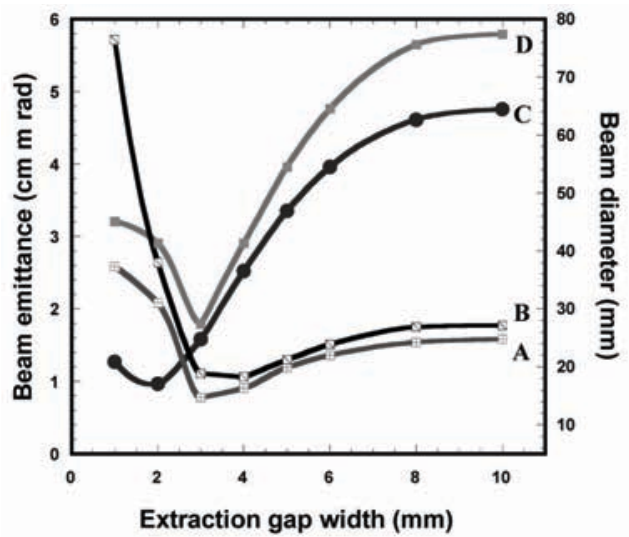

FIG. 7: Influence of the extraction gap width on the beam emittance (C/spherical, D/Pierce) and beam diameter for both (A/spherical and B/ Pierce).

the plasma boundary and the extraction electrode, where the axial potential changes rapidly. In this region, the electrons formed by ion impact on residual gas atoms will always be pulled out of the ion beam. Therefore, in this region space charge repulsion between the ions is important as soon as the ion current density becomes sufficiently large. On the other hand, in a freely drifting ion beam (no acceleration potential applied) space charge compensation will automatically work and therefore space charge repulsion is not considered there. The influence of space charge on the ion beam envelope through the extraction region of ion source was investigated. Space charge was compensated in the extraction region for ion currents between micro amps and milliamps. The same curvature radius for the concave plasma meniscus with $4 \mathrm{~mm}$ was assumed. The voltage applied to the plasma-electrode $V_{\text {plasma }}=+5 \mathrm{kV}$, the extraction electrode $V_{\text {ext }}=-4000 \mathrm{~V}$. It was found that space charge has no influence on the ion beam envelope at currents of micro amps. The space charge started to have a clear influence on the ion beam envelope at currents of $10^{-4}$ A (Fig. 8 and 9). Space charge depends on the geometry of the electrodes, applied potentials and ion current. Therefore, the change of the ion current has a clear influence where other parameters were fixed. The space charge force 


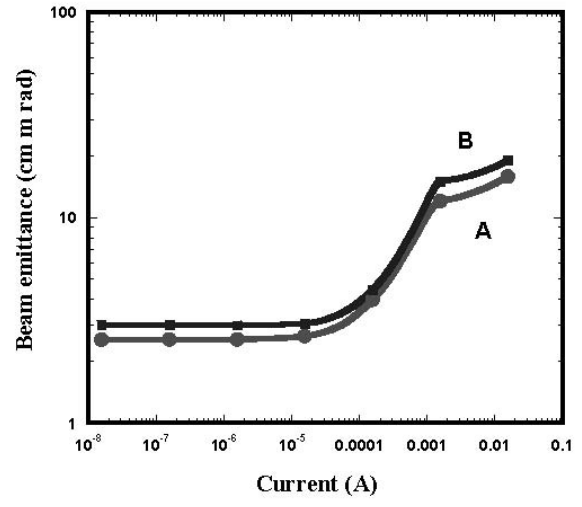

FIG. 8: Influence of current on beam diameter for spherical (A) and Pierce (B).

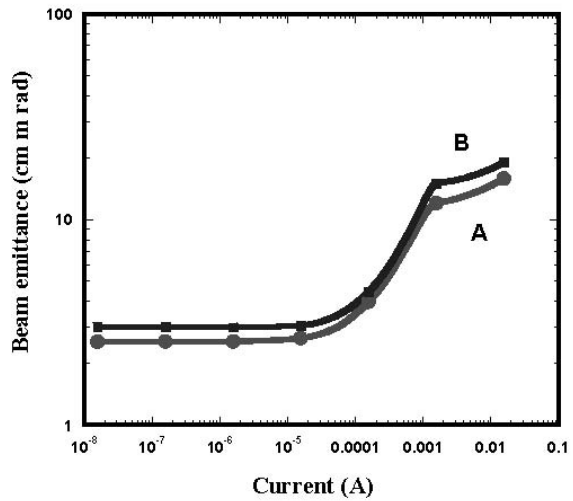

FIG. 9: Influence of current on beam emittance for spherical (A) and Pierce (B). acts as a diverging force because particles of the same charge repel each other.

\section{Conclusion}

In this work, the simulation process using SIMION computer program is used to study the influence of the cathode shape (Pierce electrode, spherical electrode with cylindrical inside canal, spherical with Pierce inside canal (used for ion the beam extraction system) on both beam diameter and beam emittance. The simulation process is made with varying extraction gap, extraction voltage and space charge effect (ion beam current). This study shows that the appropriate cathode shape is the spherical with Pierce inside canal which yields ion beam with minimum diameter and emittance.
[1] M. Turek et al; Vacuum 78; 649-654 (2005).

[2] R.G. Wilson and G.R.Brewer; "Ion Beams with Applications to Ion Implantation", John Wiley and Sons, New York (1973).

[3] H. Wituschek; Rev. Sci. Instrum., 63, 2785 (1992).

[4] J.Ishikawa; J. Appl. Phys.; 53, 6018 (1982).

[5] A. Zelenak; Rev. Sci. Instrum; 75, 5 (2004).

[6] J.R. Pierce; J. Appl. Phys; 11, 548 (1940).

[7] A.T. Forrester, "Large Ion Beams Fundamental of Generation and Propagation"; John Wiley and Sons, New York (1988).

[8] I. Langmuir and K.R.Blodgett; Phys. Rev.; 24, 49 (1924).

[9] Jr.S. Humphries; "Charged Particle Beams"; John Wiley and Sons; New York, 263 (1990).
[10] Jr.S. Humphries, "Principle of Charged Particle Acceleration"; John Wiley and Sons; New York (1999).

[11] HP. Winter, Production of Multiply Charged Heavy Ions, in Lecture notes in Physics, Vol. 83, ed. K. Bethge, Springer, Berlin 1978, 2-80.

[12] D.A. Dahl; SIMION 3 D Version 7.0 User's Manual INEEL95/0403, Idaho National Engineering and Environmental Laboratory; ID 83415 (2000).

[13] D.A. Dahl; J.E. Delmoreand A.D. Appelhans; Rev. Sci. Instrum; 61, 607 (1990).

[14] T.S. Green, IEEE, Nucl. Sci. NS. 23, 918-925 (1976). 